\title{
End-tidal carbon dioxide in predicting volume responsiveness
}

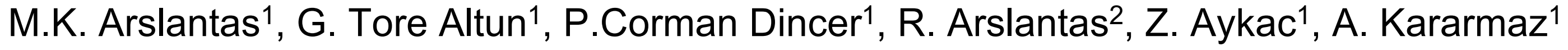 \\ ${ }^{1}$ Marmara University, School of Medicine, Department of Anaesthesiology - Istanbul (Turkey), \\ ${ }^{2}$ Health Sciences University Kartal Dr. Lutfi Kirdar Training and Research Hospital, Department of Anaesthesiology - Istanbul (Turkey)
}

\section{Background and Goal of Study:}

Stroke volume or cardiac output (CO) should be measured to assess the response to the passive leg raising test $(P L R)(1)$. However, it is not always possible to measure CO. In this study, we evaluated whether end-tidal carbon dioxide partial pressure $\left(\mathrm{PetCO}_{2}\right)$ monitoring could be an alternative method instead of $\mathrm{CO}$ measurement in evaluating the response to PLR.

\section{Materials and Methods:}

40 healthy volunteers over 18 years of age were enrolled in the study. All subjects (anaesthesiologists, residents and nurse anaesthetist) were experienced in mechanical ventilation. After at least 12 hours of fasting, non-invasive mechanical ventilation with a $5 \mathrm{mmHg}$ continuous airway pressure with a face mask without causing any leakage was initiated. The subjects were asked to keep their minute volumes constant during the study; in order to achieve this, they were seated facing the mechanical ventilator and the monitor. CO measurements were done noninvasively using USCOM1A (Sydney, AUSTRALIA). CO measurements were done three times at every position (sitting, supine position with the legs $45^{\circ}$ lifted and again sitting position) and their average values were recorded. Non-invasive blood pressures, heart rate and $\mathrm{PetCO}_{2}$ measurements were recorded.
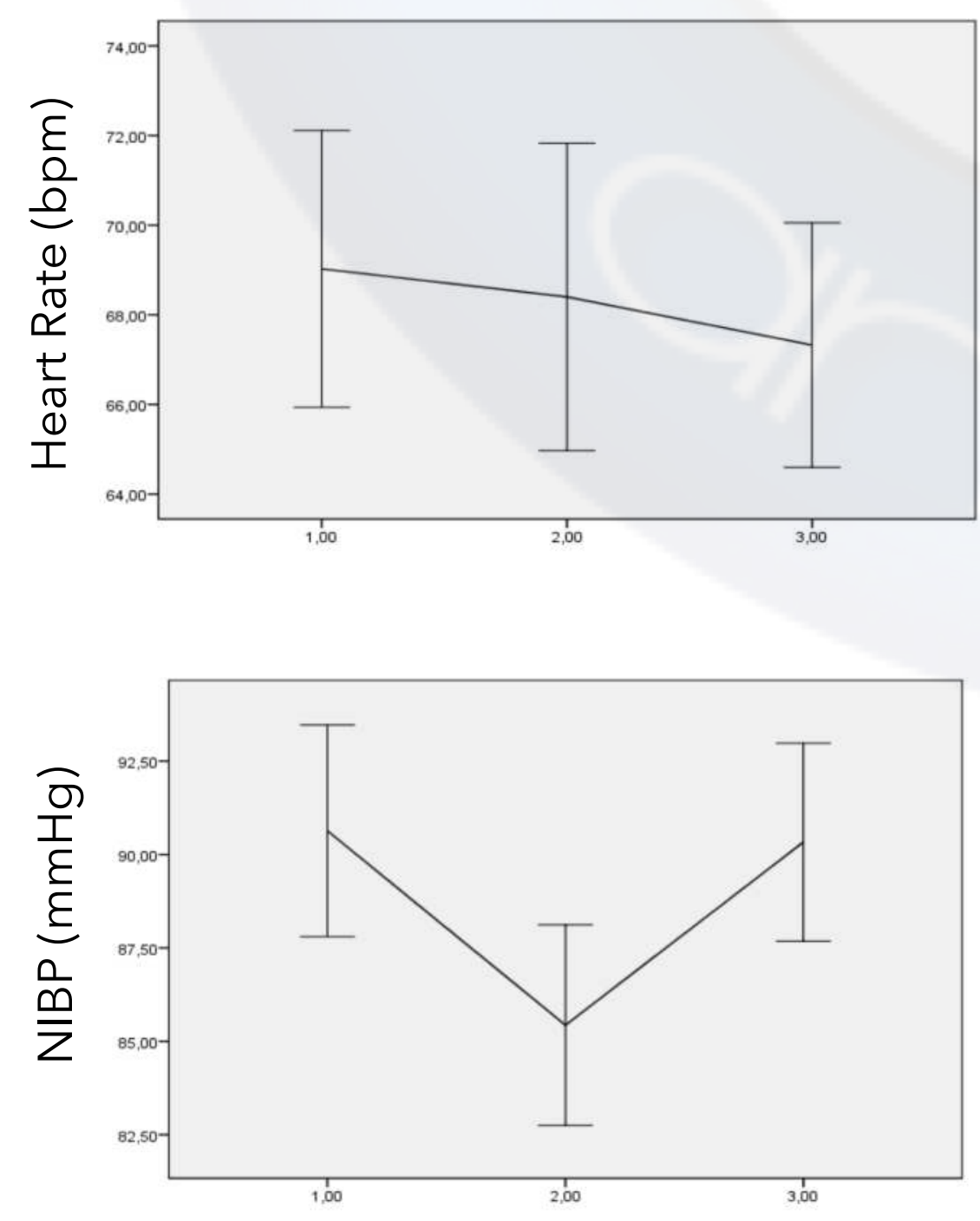
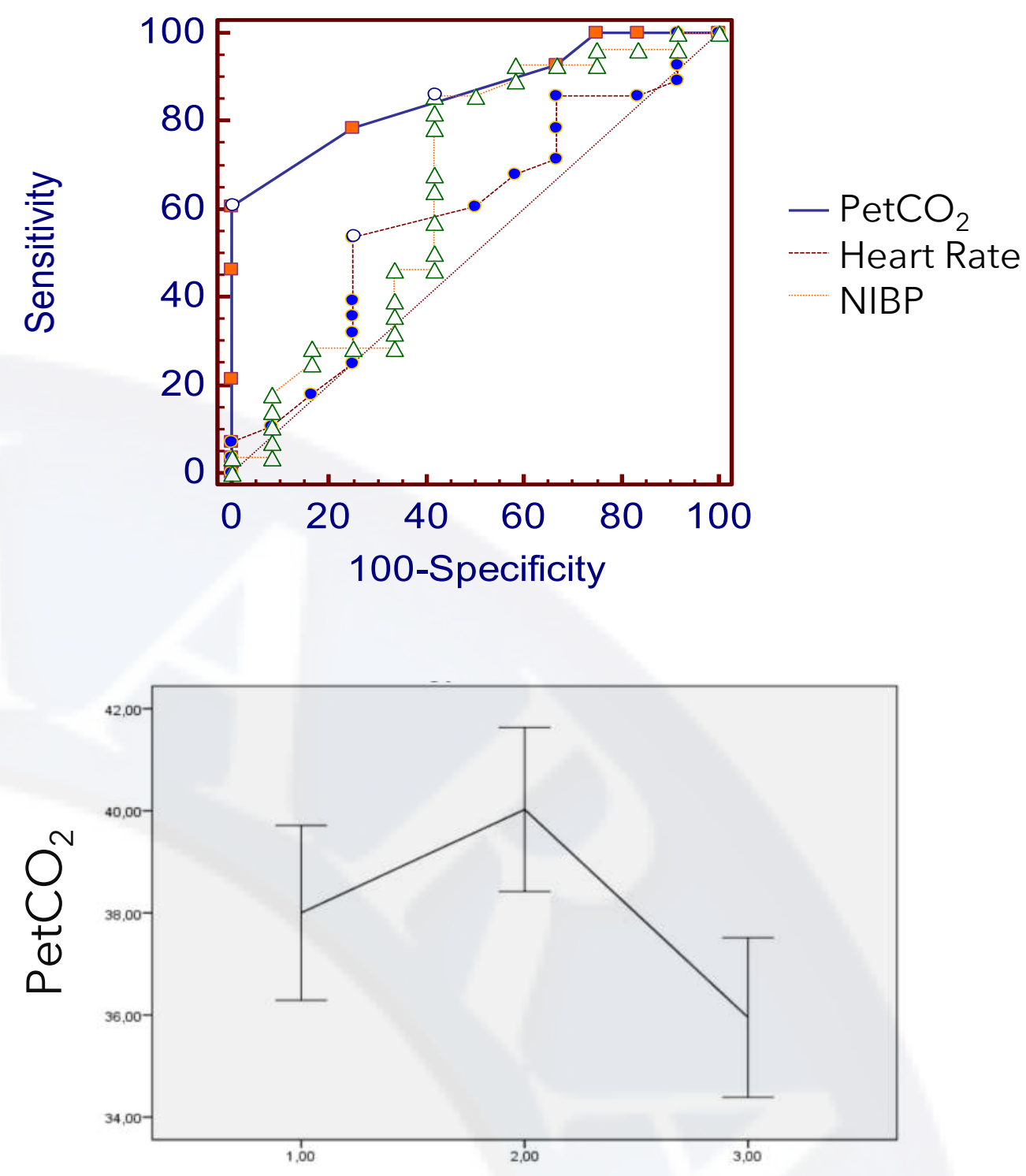

Results and Discussion:

After lifting the legs $45^{\circ}$, the CO rise was less than $10 \%$ in 12 subjects $(30 \%)$ and more than $10 \%$ in 28 subjects $(70 \%)$. No correlation was found between in heart rate and MAP changes and the changes in $\mathrm{CO}$ at the leg-up position. AUC values for the $\mathrm{HR}$ and MAP were found to be statistically insignificant. The AUC constructed for showing the ability of these two parameters to detect an increase in $C O \geq 10 \%$ and the reference curve were not significantly different from 0.5 . In leg rising position, the AUC for $\mathrm{PetCO}_{2}$ was $0.86 \pm 0.05(95 \%, \mathrm{Cl} 0.7-0.9)$ and it was statistically significant $(p<0.0001)$. The sensitivity and specificity for $\mathrm{PetCO}_{2}$ in detecting $10 \%$ change in $\mathrm{CO}$ was 69 and $75 \%$, respectively. Bootstrap method showed that the increase in PetCO $\mathrm{O}_{2}$ in between -1 and 2 was the gray zone.

\section{Conclusion:}

Similar changes in $\mathrm{CO}$ and $\mathrm{PetCO}_{2}$ values during PLR in spontaneously breathing healthy volunteers were observed. It is found that a $2 \mathrm{mmHg}$ increase in $\mathrm{PetCO}_{2}$ could be associated with an increase of $\geq 10 \%$ in $\mathrm{CO}$. We believe that $\mathrm{PetCO}_{2}$ can be used as an alternative to $\mathrm{CO}$ measurement if conditions of respiration and carbon dioxide production are fixed. 